\title{
Frequency of Neurological Disorders and Related EEG Finding in a Tertiary Care Hospital of Karachi
}

\author{
Danish Mohammad1', Syeda Saba Zaidi², Bina Fawad ${ }^{3}$, Muhammad Fazal Hussain Qureshi1 ${ }^{*}(0)$, \\ Zain Jawed Abubaker1, Muhammad Zubair Shaikh1, Muzna Shah', Mahira Lakhani', Sara Sadiq ${ }^{4}$ \\ ${ }^{1}$ Ziauddin University, Karachi, Pakistan \\ ${ }^{2}$ Department of Neurology, Liaquat National Hospital, Karachi, Pakistan \\ ${ }^{3}$ Department of Community Health Sciences, Ziauddin University, Karachi, Pakistan \\ ${ }^{4}$ Department of Physiology, CMH Institute of Medical Sciences, Bahawalpur, Pakistan \\ Email: ^fazalhqureshi22@gmail.com
}

How to cite this paper: Mohammad, D., Zaidi, S.S., Fawad, B., Qureshi, M.F.H., Abubaker, Z.J., Shaikh, M.Z., Shah, M., Lakhani, M. and Sadiq, S. (2019) Frequency of Neurological Disorders and Related EEG Finding in a Tertiary Care Hospital of Karachi. Journal of Biosciences and Medicines, 7 , 56-64.

https://doi.org/10.4236/jbm.2019.711005

Received: October 3, 2019

Accepted: November 4, 2019

Published: November 7, 2019

Copyright $\odot 2019$ by author(s) and Scientific Research Publishing Inc. This work is licensed under the Creative Commons Attribution International License (CC BY 4.0).

http://creativecommons.org/licenses/by/4.0/

\section{c) (i) Open Access}

\begin{abstract}
Background: Electroencephalogram (EEG) is defined as a method of detecting brain waves signifying the electrical conductivity of the brain. Globally, EEG is used to further classify neuropsychiatric disorders. Objective: To evaluate the prevalence of abnormal EEGs and related neurological disorders and it's correlation with age and gender. Methodology: A descriptive cross sectional study was conducted in Neurology department, Ziauddin hospital, Karachi, Pakistan from June 2018 to May 2019. A total of 440 individuals based on inclusion criteria were selected through Non-probability consecutive sampling. Informed consent was obtained from study participants. A self-designed structured questionnaire and EEG record were used for data collection. Data were entered and analyzed using SPSS v 20. Mean and standard deviation was calculated for numerical variable and frequency and percentages were calculated for categorical variable. Independent t-test and chi square was used to check association of abnormal EEG with age and gender. $P$ value of $\leq 0.05$ was considered statistically significant. Results: The mean age of study participants was $52.98 \pm 22.68$ years. There were $54.5 \%(\mathrm{n}=240)$ males. Approximately $45.2 \%(\mathrm{n}=199)$ participants had predisposing condition which can lead to abnormal EEG. EEG records of $39.8 \%(n=175)$ of the patients was normal while 60.2\% $(\mathrm{n}=265)$ had abnormal records. Diffuse neuronal dysfunction consistent with encephalopathy was found in $45.2 \%$. Mild neuronal dysfunction consistent with encephalopathy accounted for $33.5 \%$ of cases. Significant difference was found in Abnormal EEG among different age group $(p=0.01)$. Chi square shows an association between ab-
\end{abstract}


normal EEG and male gender $(\mathrm{p}=0.025)$. Conclusion: EEG plays a vital role in the diagnosis of neuropsychiatric conditions in developing countries. EEGs can further help to determine the relationship of different neuropsychiatric conditions and can help in early diagnosis and better prognosis.

\section{Keywords}

Neurological Disorders, EEG, Association

\section{Introduction}

Electroencephalogram (EEG) is defined as a method of detecting brain waves signifying the electrical conductivity of the brain using small metal electrodes recorded as wavy lines on EEG recording paper [1]. EEG waves consist of alternating excitatory and inhibitory synaptic potentials generated in the pyramidal cells of the cerebral cortex [2]. EEG waves are classified as following: Alpha $(\alpha)$, Beta $(\beta)$, Theta $(\theta)$, and Delta $(\delta)$. Alpha waves are seen at frequencies between 18 and 30 cycles/sec. They are found predominantly in healthy adults while awake or in resting states of cerebration. Beta waves are seen at frequencies between 14 to $80 \mathrm{cycles} / \mathrm{sec}$ and are found when a person is directed towards specific mental activity. Theta waves are seen at frequencies between 4 and $7 \mathrm{cy}$ cles/min. They are predominantly found in children, but can also be found during states of emotional stress in adults. They are also markers of degenerative brain disorders. Delta waves are seen at frequencies below $3.5 \mathrm{cycles} / \mathrm{sec}$. They are markers of deep sleep and organic brain disease [3].

Physicians have declared EEG as a gold standard diagnostic test for seizures and epilepsy [4]. Globally, EEG is used to further classify neuropsychiatric disorders. Furthermore, studies have focused on analysing the significance of different algorithms and techniques for EEG. Khanna et al. examines microstates in resting state EEG for diagnosis of multiple neurological disorders such as schizophrenia, dementia, and depression [5]. In addition, wavelength based EEG have been established, as the most effective automated method for seizures and epilepsy [6]. Moreover, physicians are trying to prove brain death with the help of EEG reports [7]. Multiple methods have been developed to diagnose encephalitis using EEG [8].

Even though EEG is important for diagnosis but limited studies have been carried out in Pakistan. Most of the studies conducted have been used to analyse better treatment and diagnosis of disorder [9] [10] [11] [12]. Very few articles assess the demographic and etiological trend of disease [13]. However, there is minimal research on observation of the basic trends of EEG and the use of EEG in our setup. Therefore, the objective of this study is to evaluate the prevalence of abnormal EEGs and related neurological disorders and it's correlation with age and gender. 


\section{Methodology}

A descriptive cross sectional study was conducted in neurological department of Ziauddin Hospital. The study duration was one year i.e. June 2018 to May 2019. The actual sample size was 384 individuals, which was calculated by using the standard formula for calculating sample size on the basis of prevalence. Prevalence was taken at $50 \%$ because no relevant data was available. The bound of error was $5 \%$ with a $95 \%$ confidence interval. The sample size was inflated to exclude non-response and poorly filled questionnaires.

Non probability consecutive sampling technique was used. Inclusion criteria consisted of all patients referred for EEG to neurology department while those were excluded who either refused to give consent for participation or from Paediatric age group or Unconscious patients or patients with altered mental status. A self-designed questionnaire was used for collection of data. Questionnaire contained information including demographics (age, gender), pertinent history (drug, family, substance abuse), source of referral, activation procedure if performed during EEG, EEG findings, provisional diagnosis made by physician referring for EEG, Conclusion and then EEG correlation with the provisional diagnosis. The EEG records were reviewed. Socio demographic data, clinical data and the neurologist's comments on the EEG recordings were extracted and recorded on the proforma. Consent was obtained from every participant. Ethical approval was taken from Institutional review board before conducting study.

The data was analysed using Statistical Package for the Social Sciences (SPSS) version 20. Quantitative variables were presented as mean and standard deviation while qualitative variables were presented as frequency and percentages. Outcome variable was EEG correlation with the provisional diagnosis. Independent $\mathrm{t}$-test and chi square was calculated to check association of abnormal EEG with age and gender.

\section{Results}

A total of 440 patients referred for EEG were included in the study. Majority 240 (54.5\%) of them were males, mean age was $52.98 \pm 22.68$ years. The age distributions of the patients were as follows: Majority, 196 (44.5\%) were in the age bracket 25 - 64 years, 172 (39.9\%) were 65+ years, 69 (16.3\%) were between 15 24 years. About $98 \%$ of participants have no history of addictions. Referrals from neuro department were $86.1 \%$ while the rest of the $13.9 \%$ were from medicine, surgery and other departments as shown in Table 1.

About 44\% (191) participants were on medications which include Lerace, Epival, Rivotril, midazolam, Keppra, phenytoin, Valium and hitop. Looking over the predisposing conditions that can lead to abnormal EEG findings, approximately 45.2\% (199) participants were prone, as shown in Figure 1.

The EEG diagnosis based on the interpretation of the EEG records by the neurologists showed that 175 (39.8\%) of the patients had normal records while $265(60.2 \%)$ had abnormal records. The breakdown of the abnormal records 


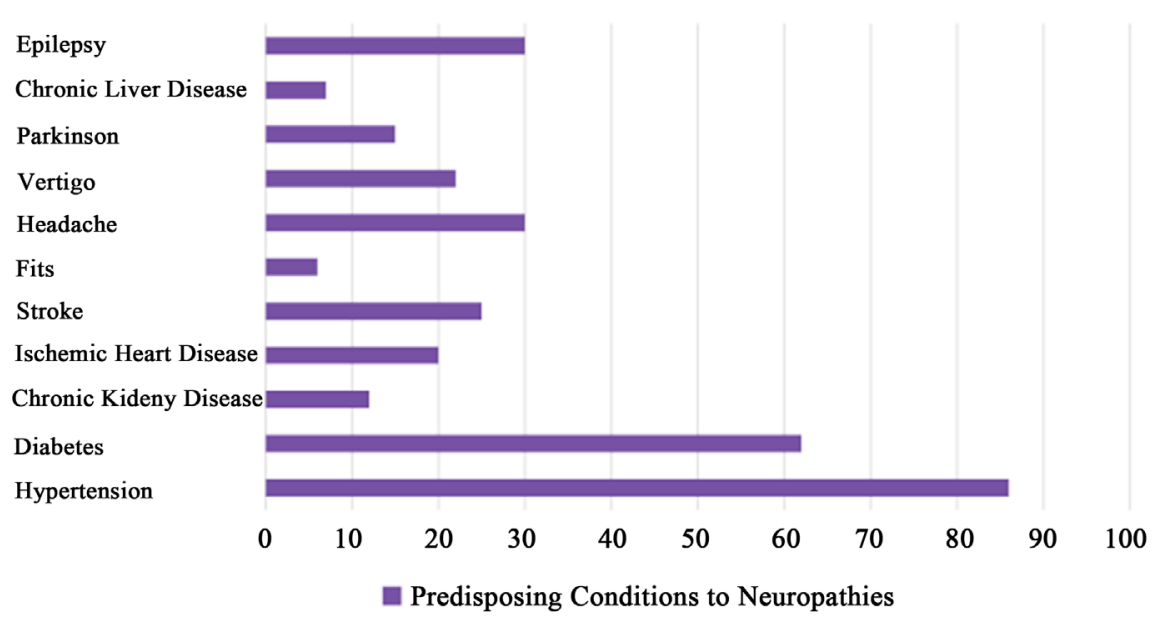

Figure 1. Predisposing condition I sample which can lead to abnormal EEG findings.

Table 1. Socio-demographics of participants.

\begin{tabular}{|c|c|c|c|c|c|c|}
\hline & \multicolumn{2}{|c|}{ Male } & \multicolumn{2}{|c|}{ Female } & \multicolumn{2}{|c|}{ Combined } \\
\hline & $\mathbf{N}$ & $\%$ & $\mathbf{n}$ & $\%$ & $\mathbf{n}$ & $\%$ \\
\hline Youth (15 - 24) & 36 & 18 & 36 & 18 & 72 & 16.36 \\
\hline Adults (25 - 64) & 106 & 44.16 & 90 & 45 & 196 & 44.54 \\
\hline Senior $(65+)$ & 98 & 40.83 & 74 & 37 & 172 & 39.09 \\
\hline \multicolumn{7}{|c|}{ Family History } \\
\hline Positive & 01 & 0.4 & 01 & 0.4 & 02 & 0.5 \\
\hline Negative & 239 & 99.6 & 199 & 99.6 & 438 & 99.5 \\
\hline \multicolumn{7}{|c|}{ Addiction History } \\
\hline None & 232 & 96.7 & 200 & 100 & 432 & 98.2 \\
\hline Alcohol & 5 & 2.1 & 0 & 0 & 5 & 1.1 \\
\hline Smoking & 3 & 1.3 & 0 & 0 & 3 & 0.7 \\
\hline \multicolumn{7}{|c|}{ Referral Department } \\
\hline Neuro & 213 & 88.8 & 166 & 83 & 379 & 86.1 \\
\hline Others & 27 & 11.3 & 34 & 17 & 61 & 13.9 \\
\hline
\end{tabular}

showed that diffuse neuronal dysfunction consistent with encephalopathy accounted for $27.2 \%$ while Mild neuronal dysfunction consistent with encephalopathy accounted for $20.2 \%$ of cases. EEG findings resulted in a change of diagnosis in the majority of patients (Table 2).

Further, to check association of abnormal EEG with age independent sample $t$ test was used, which shows strong significant difference with $\mathrm{p}$ value less than 0.01. Chi-Square was applied to check significance among abnormal EEG and gender which shows significant relationship with males with $\mathrm{p}$ value to 0.025 . 
Table 2. EEG procedure and diagnosis.

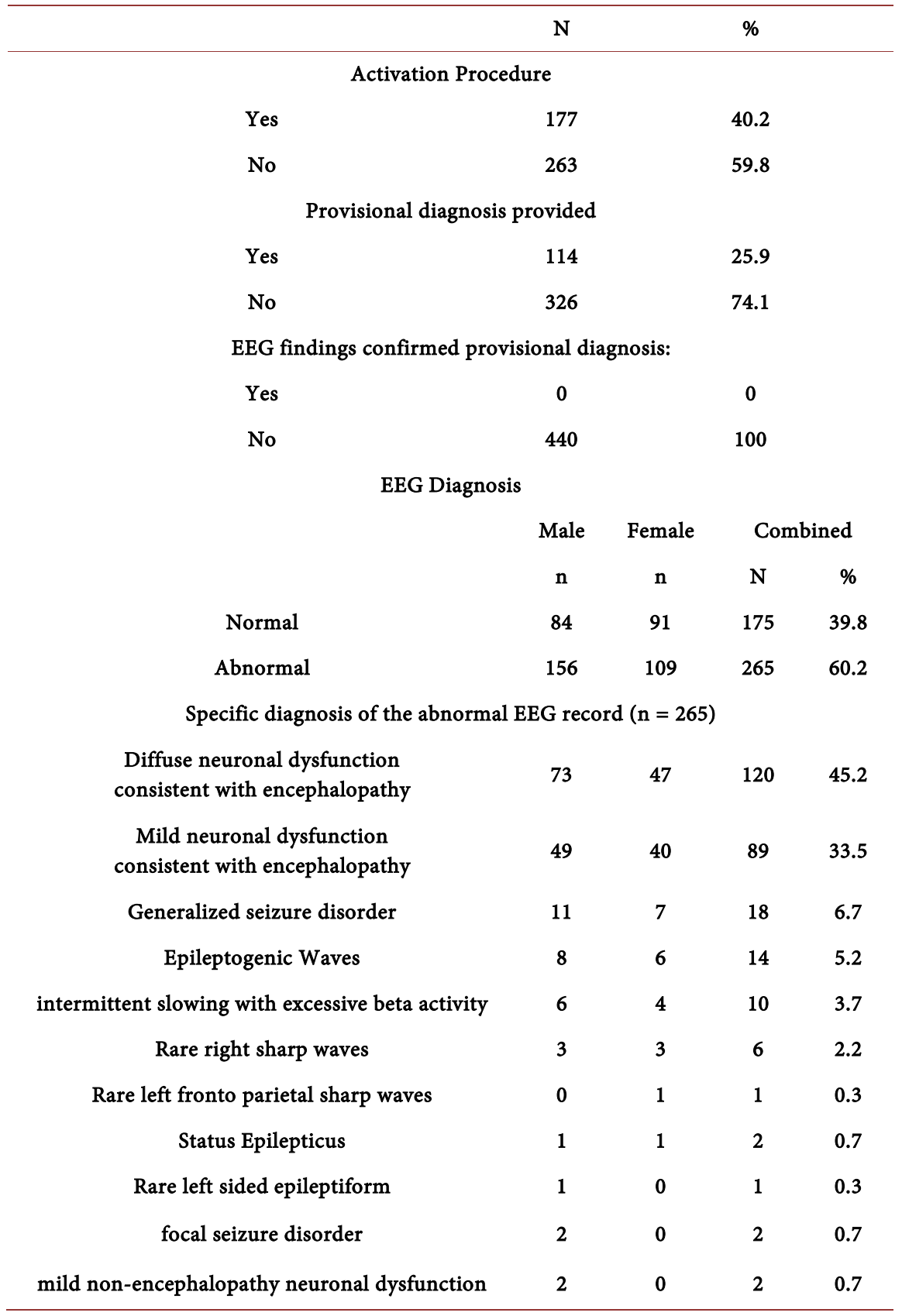

\section{Discussion}

The outcome of our study seems predictable with a couple interesting findings. The study shows that in our particular setup $60.2 \%$ of EEGs were considered abnormal whereas $39.8 \%$ were denounced normal. Initially, more seizure disorders were predicted before the study. However, after the study we see that the majority of patients suffer diffused or mild neuronal dysfunction with encephalopathy leading up to $47.4 \%$ of the cases. The mean age for patients using EEG came out to be approximately 53 years. Further observations point out that the main predisposing factors patients have are diabetes and hypertension and $74 \%$ 
of the time no provisional diagnosis is mentioned before a study.

More abnormal EEGs were the outcome. Our study shows that $60.2 \%$ of patients having abnormal readings. Compared to other studies it seems to be the norm to have more abnormal EEGs than normal ones. One Nigeria based study showed 56 percent of patients having epileptiform activity [14]. On the other hand, Nigeria seemed to have $32.4 \%$ abnormal EEGs [15]. Even though this particular study is out of the norm one thing to know is that normal EEGs do not rule out any epileptic disorders. For example, a study by Ahmed et al. showed that $35.6 \%$ of epileptic patients showed normal reading [16]. This reinforces the fact that even though the abnormal reading for our results were $60.2 \%$, there could be several more patients in the normal readings with seizure disorders.

The mean age for our study seemed to be older than the normal. Majority of the patients were between the age brackets 25 - 64 which comprise of $44.54 \%$ of patients. In addition to this, $39.9 \%$ of patients were above 65 in our setup. This finding is unusual because most EEG studies seem to have patients younger than 30. For example a study by Ahmed et al. suggests that the majority of the patients were predominantly below 20 years of age [16]. Another study from Kathmandu suggests that 50 percent of patients ranged from 11 - 30 years [17]. This may be because most of the patients in these studies were found to have seizure disorder, whereas our particular setup had more patients with neuronal dysfunction and encephalopathy. This points out that our setup may simply have less seizure disorders which is why we have more people ranging 25 and above.

Out of the abnormal EEGs the main diagnosis seemed to be either $45.2 \%$ diffuse neuronal dysfunction with encephalopathy or 33.5\% mild neuronal dysfunction with encephalopathy. These stats show that few patients in our setup had seizure disorders. However, several other studies have more seizure disorder patients during EEG. Not much research has been conducted on seizure disorders in Pakistan. Only one study looks at the aetiology of status epilepticus in patients [18]. It states that $14 \%$ of the patients had status epilepticus because of progressive encephalopathy. This correlates with our set up because it is possible that a small percentage of our patients are at risk for status epilepticus even though the patients studied in that research were children. More importantly, the results suggest that rather than using EEG for seizure disorders our population needs a higher focus on why there is neuronal dysfunction with encephalopathy. Johnson et al. states that encephalopathy is a state of unconsciousness that can have multiple causes such as the following: metabolic toxicity, drug toxicity, structural dysfunction of the brain or infection [19]. Based on each patient's history, there needs to be an effort to figure out the cause that makes this finding prevalent. In addition, the finding of neuronal dysfunction can suggest further risk factors such as Alzheimer's disease. For example, one longitudinal study stated that after following patients with mild neuronal dysfunction for three years EEG showed that some patients developed Alzheimer's disease with more theta/delta waves [20]. In this study EEG was used as a prognostic tool, therefore 
this means that it can be used as a prognostic tool in our setup as well.

Hypertension and diabetes are the main predisposing factors patients come with before an EEG, therefore we can expect certain changes in EEG when managing a patient. Like current study reported majority of patients were having diabetes and hypertension. There is not much known about hypertension and its effects on EEG readings. However, according to an article Inova et al. we can expect decreased alpha rhythms from patients with hypertension [21]. Furthermore, another study suggests that in stressed hypertensive patients there can be reduced theta waves in the readings [22]. As a result, these studies show what changes we can expect for a predisposing hypertensive patient. In diabetes one can consider that factor as a contributor to metabolic encephalopathy. In one case report, hypoglycaemia showed signs of theta waves in a 45 year old patient [23]. This is important because these are diagnostic signs you can see in certain patients with diabetes as a predisposing factor. Hypoglycaemia can be related to mild neuronal dysfunction with encephalopathy [19]. This fact can allow us to come up with differentials when doing an EEG study on a diabetic.

In our setup $74 \%$ of the time no provisional diagnosis is stated. Furthermore, in our study the provisional diagnoses have never been confirmed by the EEG. Therefore, doctors must ask what they are screening for. EEG is considered a great screening tool with higher specificity when it comes to epilepsy [14] [15]. However, many researchers state that doctors do not know how to use EEG. One researcher states that non specialist try to use it as a diagnostic tool when clinicians should be using it as an aid in differentials [24]. In one study it stated that $26 \%$ of requests from physicians for EEG were considered inappropriate [25]. These facts state that maybe EEG is being overused. It does not mean that we should completely abstain from EEGs, rather we should take into consideration finances, need and proficiency before ordering one.

This study conducted gives us an idea on what to focus on and what to look out for. This study shows that in our particular setup older patients, usually hospitalized, will show probable signs of neuronal dysfunction with encephalopathy. Further, studies suggest that younger patients under 30 will probably show particular signs of seizure disorders. Not every case will be perfect, but these trends are something we can expect. Furthermore, we know in our hospital setup we should expect more neuronal dysfunction with encephalopathy. As a result, we can better management and treatment by looking into what is the cause of this particular finding. We would have to do a further study and increase the sample specifically for these patients. We also know that most patients that come in for an EEG are hypertensive or diabetic. Therefore, with this study we should look into what particular signs are specific for these predisposing factors. We can use this information to further study these patients on EEG. Finally, we see that EEGs are not matching the provisional diagnosis. Studies must be conducted to see whether the EEGs that are being ordered actually aid in the management and treatment of the patient or we can do away with them while reducing the cost of healthcare for our patients. 
Limitation for the current study was that data was from one particular hospital setup. Similar studies should be done in different hospitals all over Pakistan to get better and more accurate results. Another limitation was the small sample size. It should be done on a larger scale so there is more data to analyse particularly correlations amongst causes and effects. There should also be comparison studies amongst provinces to see any particular ethnic variations.

\section{Conclusion}

EEG still plays a vital role in the diagnosis of neuropsychiatric conditions in developing countries. EEGs can further help to determine the relationship of different neuropsychiatric conditions and can help in early diagnosis and better prognosis.

\section{Conflicts of Interest}

The authors declare no conflicts of interest regarding the publication of this paper.

\section{References}

[1] Mayo Clinic (2018) EEG (Electroencephalogram). https://www.mayoclinic.org/tests-procedures/eeg/about/pac-20393875

[2] Costanzo, L.S. (2015) BRS Physiology. 7th Edition, Wolters Kluwer, Netherlands, $318 \mathrm{p}$.

[3] Hall, J.E. (2011) Guyton and Hall Textbook of Medical Physiology. 13th Edition, \ Saunders, Philadelphia, PA, 1145 p.

[4] Rosenow, F., Klein, K.M. and Hamer, H.M. (2015) Non-Invasive EEG Evaluation in Epilepsy Diagnosis. Expert Review of Neurotherapeutics, 15, 425-444. https://doi.org/10.1586/14737175.2015.1025382

[5] Khanna, A., Pascual-Leone, A., Michel, C.M. and Farzan, F. (2015) Microstates in Resting-State EEG: Current Status and Future Directions. Neuroscience and Biobehavioral Reviews, 49, 105-113. https://doi.org/10.1016/j.neubiorev.2014.12.010

[6] Faust, O., Acharya, U.R., Adeli, H. and Adeli, A. (2015) Wavelet-Based EEG Processing for Computer-Aided Seizure Detection and Epilepsy Diagnosis. Seizure, 26, 56-64. https://doi.org/10.1016/j.seizure.2015.01.012

[7] Szurhaj, W., Lamblin, M.D., Kaminska, A. and Sediri, H. (2015) EEG Guidelines in the Diagnosis of Brain Death. Neurophysiologie Clinique/ Clinical Neurophysiology, 45, 97-104. https://doi.org/10.1016/j.neucli.2014.11.005

[8] Sutter, R., Kaplan, P.W., Cervenka, M.C., Thakur, K.T., Asemota, A.O., Venkatesan, A., et al. (2015) Electroencephalography for Diagnosis and Prognosis of Acute Encephalitis. Clinical Neurophysiology. Official Journal of the International Federation of Clinical Neurophysiology, 126, 1524-1531. https://doi.org/10.1016/j.clinph.2014.11.006

[9] Ahmad, M.A., Ayaz, Y., Jamil, M., Omer Gillani, S., Rasheed, M.B., Imran, M., et al. (2015) Comparative Analysis of Classifiers for Developing an Adaptive Computer-Assisted EEG Analysis System for Diagnosing Epilepsy. BioMed Research International, 2015, Article ID: 638036. https://doi.org/10.1155/2015/638036

[10] Hussain, L., Aziz, W., Alowibdi, J.S., Habib, N., Rafique, M., Saeed, S., et al. (2017) 
Symbolic Time Series Analysis of Electroencephalographic (EEG) Epileptic Seizure and Brain Dynamics with Eye-Open and Eye-Closed Subjects during Resting States. Journal of Physiological Anthropology, 36, 21. https://doi.org/10.1186/s40101-017-0136-8

[11] Shahnaz, S., Sher, K. and Abdul Sattar, R. (2014) Clinical and EEG Characteristics of Juvenile Myoclonic Epilepsy. Pakistan Journal of Medical Sciences, 30, 12-15. https://doi.org/10.12669/pjms.301.4465

[12] Marchi, N., Granata, T. and Janigro, D. (2014) Inflammatory Pathways of Seizure Disorders. Trends in Neurosciences, 37, 55-65.

https://doi.org/10.1016/j.tins.2013.11.002

[13] Hussain, S. and Tarar, S.H. (2015) Febrile Seizrues: Demographic, Clinical and Etiological Profile of Children Admitted with Febrile Seizures in a Tertiary Care Hospital. The Journal of the Pakistan Medical Association, 65, 1008-1010.

[14] Aina, O.F., Malomo, I.O., Ladapo, H.T. and Amoo, I.G. (2004) One Year of EEG Unit at Psychiatric Hospital, Yaba, Lagos. Nigerian Postgraduate Medical Journal, 11, 212-214.

[15] Ajiboye, P.O., Abiodun, O.A. and Ogbebor, A.I. (2017) An Investigation of the Patterns and Outcomes of Electroencephalographic (EEG) Recording Requests in the Management of Neuropsychiatric Disorders in a Teaching Hospital in Nigeria. $A f$ rican Health Sciences, 17, 852-858. https://doi.org/10.4314/ahs.v17i3.28

[16] Ahmed, M.H. and Obembe, A. (1991) Electroencephalographic Abnormalities in 351 Nigerians with Epilepsy. West African Journal of Medicine, 10, 216-221.

[17] Shrestha, R., Pradhan, S.N., Sharma, S.C., Shakya, K.N., Karki, D.B., Rana, B.B., et al. (2004) A Study of the First 350 Cases Referred for EEG in Kathmandu Medical College Teaching Hospital. Kathmandu University Medical Journal, 2, 24-27.

[18] Uzair, M., Ibrahim, A., Zafar, F. and Sultan, T. (2019) Etiology and Outcomes of Convulsive Status Epilepticus in Children. Pakistan Journal of Medical Sciences, 35, 620-623. https://doi.org/10.12669/pjms.35.3.120

[19] Johnson, E.L. and Kaplan, P.W. (2019) Chapter 4-Clinical Neurophysiology of Altered States of Consciousness: Encephalopathy and Coma. In: Levin, K.H. and Chauvel, P., Eds., Handbook of Clinical Neurology, Elsevier, Amsterdam, 73-88. https://doi.org/10.1016/B978-0-444-64142-7.00041-2

[20] Musaeus, C.S., Nielsen, M.S. and Hogh, P. (2019) Altered Low-Frequency EEG Connectivity in Mild Cognitive Impairment as a Sign of Clinical Progression. Journal of Alzheimer's Disease, 68, 947-960. https://doi.org/10.3233/JAD-181081

[21] Ivanova, O.M. and Ivanova, M.A. (2018) The Significance of EEG in the Evaluation of Arterial Hypertension Treatment. Zhurnalnevrologiiipsikhiatriiimeni SS Korsakova, 118, 72-74. https://doi.org/10.17116/jnevro201811808172

[22] Wang, H.X., Li, S.Z., Lu, X.Y. and Zhang, L. (2015) Relationship between Electroencephalogram and Perceived Stress in Essential Hypertension Patients. Acta Academiae Medicinae Sinicae, 37, 650-655.

[23] Ryu, H.U., Lee, S., Shin, B.S. and Kang, H.G. (2019) Different Patterns of Electroencephalography during Hypoglycemia in Type 1 Diabetes Mellitus: A Case Report. Medicine, 98, e16483. https://doi.org/10.1097/MD.0000000000016483

[24] Smith, D., Bartolo, R., Pickles, R.M. and Tedman, B.M. (2001) Requests for Electroencephalography in a District General Hospital: Retrospective and Prospective Audit. British Medical Journal, 322, 954-957. https://doi.org/10.1136/bmj.322.7292.954

[25] Pearce, K.M. and Cock, H.R. (2006) An Audit of Electroencephalography Requests: Use and Misuse. Seizure, 15, 184-189. https://doi.org/10.1016/j.seizure.2006.01.003 